\title{
Immunohistochemical Localization of Indole-3-Acetic Acid During Induction of Adventitious Root Formation from Cotyledon Explants of Walnut
}

\author{
Ningguang Dong, Qingmin Wang, Junpei Zhang, and Dong Pei ${ }^{1}$ \\ Laboratory of Tree Breeding and Cultivation, State Forestry Administration, Research Institute of \\ Forestry, Chinese Academy of Forestry, Beijing 100091, P.R. China
}

\begin{abstract}
AdDitional INDEX wORDs. auxin, immunolabeling, Juglans regia, rooting, vascular differentiation
Abstract. Cotyledon explants of walnut (Juglans regia) have been shown to generate adventitious roots on growth regulator-free medium. The spatial distribution of endogenous indole-3-acetic acid (IAA) and its dynamic changes during adventitious root formation were investigated using an in situ immunohistochemical approach. Before root induction, IAA signal was distributed throughout the freshly excised cotyledon explants. During provascular bundle differentiation, the IAA signal was mainly located in the provascular bundles. At the stage of annular meristematic zones formation, the IAA signal was mainly distributed in the meristematic zones and decreased in the vascular bundles and cotyledonous parenchyma. As primordia formed, the IAA signal became localized in the root primordia and gradually disappeared in the meristematic zones. In emerging roots, the IAA signal was mainly localized in the root cap and root meristem. These results suggest that accumulation of IAA in the provascular bundles may induce vascular differentiation and the increase in IAA through meristematic zones may be responsible for the adventitious root formation from walnut cotyledons. The direct evidence presented here indicates that IAA accumulated in the meristematic zones is not the sole signal needed to induce adventitious root.
\end{abstract}

The relationship between auxin and adventitious root formation has been studied for many years. Indole-3-acetic acid plays a central role in adventitious rooting and was the first plant hormone used to stimulate the rooting of cuttings (Cooper, 1935). To date, several studies have addressed the role of auxin in adventitious root formation of plants such as arabidopsis [Arabidopsis thaliana (Ludwig-Muller et al., 2005)], red pepper [Capsicum annuum (Ahmad et al., 2006)], eucalyptus [Eucalyptus urophylla $\times$ E. grandis (Nourissier and Monteuuis, 2008)], and zephyr lily [Zephyranthes grandiflora (Gangopadhyay et al., 2010)]. Most of these studies focused on quantitative analysis of auxin using various analytical methods and on biological characteristics of adventitious roots after application of exogenous auxin. Although these studies have provided important information concerning the action of this hormone, the mechanism of auxin in adventitious root induction has not been clearly established. Results obtained from these studies were generally complicated by the absorption or transportation of exogenously applied auxin. Additionally, the concentration of auxin at the target site, rather than throughout the entire tissue, should more accurately reflect its active level.

With the application of immunology in botany, it has become possible to detect in situ auxin in plant tissues. Immunohistochemical localization techniques have been used previously in maize [Zea mays (Shi et al., 1993)], peanut [Arachis hypogaea (Moctezuma, 1999; Moctezuma and Feldman, 1999)], embryos of sunflower [Helianthus annuus (Thomas et al., 2002)],

Received for publication 13 July 2011. Accepted for publication 23 Aug. 2011. This work was supported by the National Natural Science Foundation of China (No. 31171933) and the Commonweal Special Foundation of State Forestry Administration of China (No. 201004048).

We sincerely thank Dr Zhixia Hou (College of Food Science and Nutritional Engineering, China Agricultural University, China) for their kind help with experiment methods and the equipment.

${ }^{1}$ Corresponding author. E-mail: peidonggu@163.com. arabidopsis (Aloni et al., 2003; Avsian-Kretchmer et al., 2002), the shoot apices of strawberry [Fragaria Xananassa (Hou and Huang, 2005)], and tobacco [Nicotiana tatacum (Chen et al., 2010)]. However, many aspects of IAA distribution and its action mechanism in adventitious root formation remain unknown.

In walnut, cotyledon explants from the region of attachment to the embryonic axis have been shown to generate adventitious roots in the absence of exogenous growth regulators (Ermel et al., 2000; Gutmann et al., 1996; Jay-Allemand et al., 1991). This system is very suitable for the study of the internal dynamics of endogenous IAA because no exogenous auxin is required for the induction of adventitious roots. Because no exogenous auxin is added, we hypothesized that culture in vitro leads to redistribution, production, or release of endogenous auxin from inactive forms within the cotyledon explants. This hypothesis is impossible to verify by the use of conventional hormone quantification (e.g., enzyme-linked immunosorbent assay or mass spectrometry), because spatial information is lost during the extraction step. In the present study, the spatial distribution of endogenous IAA and its dynamic changes during adventitious root formation in walnut cotyledons were investigated using an immunohistochemical approach. This study provides a substantial base to understand the IAA mechanism at the cellular level during rhizogenesis.

\section{Materials and Methods}

Plant material and induction of adventitious roots. Mature seeds of walnut cultivar Jinlong 2 were obtained from the walnut germplasm repository of the Shanxi Academy of Forestry. Cotyledon explants from the region of attachment to the embryonic axis (Fig. 1) were dissected under axenic conditions as previously described (Jay-Allemand et al., 1991). These explants were induced to root in a growth regulator-free medium in the 
dark at $26^{\circ} \mathrm{C}$ for periods of over $6 \mathrm{~d}$ as described by Jay-Allemand et al. (1991) and were collected at different times of culture.

FIXATION OF SAMPLES AND PREPARATION OF SEMITHIN SECTIONS. Cotyledon explants from the region of attachment to the embryonic axis (Fig. 1) were collected at different times of development $(0,24,48,72,96,120$, and $144 \mathrm{~h}$ of in vitro culture). The explants were prefixed immediately in a $2 \%(\mathrm{w} / \mathrm{v})$ aqueous solution of 1-ethyl-3-(3-dimethylaminopropyl)-carbodiimide [EDC (Sigma, St. Louis, MO)] and post-fixed overnight in a solution containing $4 \%(\mathrm{w} / \mathrm{v})$ paraformaldehyde and $2.5 \%(\mathrm{v} / \mathrm{v})$ glutaraldehyde at $4{ }^{\circ} \mathrm{C}$. The fixed tissues were then dehydrated through a graded ethanol series, embedded in paraffin, sectioned at $8 \mu \mathrm{m}$, and submitted to the immunolocalization procedure.

INDOLE-3-ACETIC ACID IMMUNOLOCALIZATION. The immunolocalization of IAA was performed as described in Holgate et al. (1983) with slight modifications. Briefly, sections were incubated for $45 \mathrm{~min}$ in blocking solution [0.05 M Tris buffer (TBS), pH 7.6, 0.3\% (v/v) Triton X-100, 10\% (v/v) normal goat serum, and $5 \%(\mathrm{w} / \mathrm{v})$ bovine serum albumin $(\mathrm{BSA})]$ and incubated overnight at $4{ }^{\circ} \mathrm{C}$ with anti-IAA antibodies (Agdia, Elkhart, IN) diluted 1:200 in a TBS/BSA solution. Subsequently, the sections were incubated for $4 \mathrm{~h}$ at room temperature with gold-labeled goat antimouse $\mathrm{IgG}(15 \mathrm{~nm}$ in diameter) diluted 1:50 in TBS/BSA. After washing, the sections were stained with a silver staining solution $[0.1 \mathrm{M}$ citrate buffer, $\mathrm{pH}$ $3.5,1.7 \%(\mathrm{w} / \mathrm{v})$ hydroquinone, and $0.1 \%(\mathrm{w} / \mathrm{v})$ silver nitrate]. As the color developed $(15 \mathrm{~min})$ in the sections, they were rinsed with water, dehydrated, mounted, observed, and photographed.

To verify the reliability of the immunolocalization technique and specificity of the anti-IAA antibodies, three negative controls were included: no EDC prefixation, no anti-IAA antibodies, and the substitution of normal mouse serum for the anti-IAA antibodies.

Analysis of indole-3-acetic ACID Distribution. The distribution of IAA during adventitious root formation in walnut cotyledons was revealed by reddish brown silver particles in stained sections (such as Fig. 2A1). For each tissue, the number of silver particles in each of 30 visual fields in a section was counted under an oil lens $(100 \times$ objective lens, $10 \times$ ocular lens). The labeling density is presented as the number of silver particles per $100 \mu^{2}$. All treatments were repeated at least three times, and all samples were analyzed three times. Analysis of variance was performed, and significant differences between means were determined using a multiplerange test. Significance was assumed at $P<0.05$.

\section{Results}

\section{Distribution of indole-3-acetic acid in cotyledon tissue during adventitious root formation}

The process of adventitious root formation in the cotyledon explants showed the same time course of morphological and histological changes as previously described (Ermel et al., 2000; Gutmann et al., 1996; Jay-Allemand et al., 1991). The distribution of IAA in the walnut cotyledon was revealed by reddish brown silver particles. The immunological and statistical results are shown in Figs. 2 through 5.

STAGE 0: INITIAL PERIOD-COTYLEDON EXPLANTS BEFORE IN VITRO CULTURE (AT 0 H). IAA signal was detected throughout freshly excised cotyledon explants before culture (Figs. 2A, 2A1, and 2A2). The IAA level in the provascular cells was slightly higher

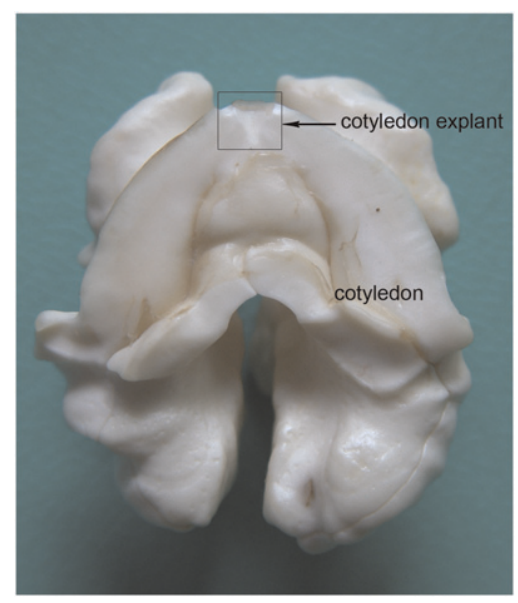

Fig. 1. Freshly excised walnut cotyledon. After removal of the embryonic axis, cotyledon explants were dissected under axenic conditions and then cultured in vitro.
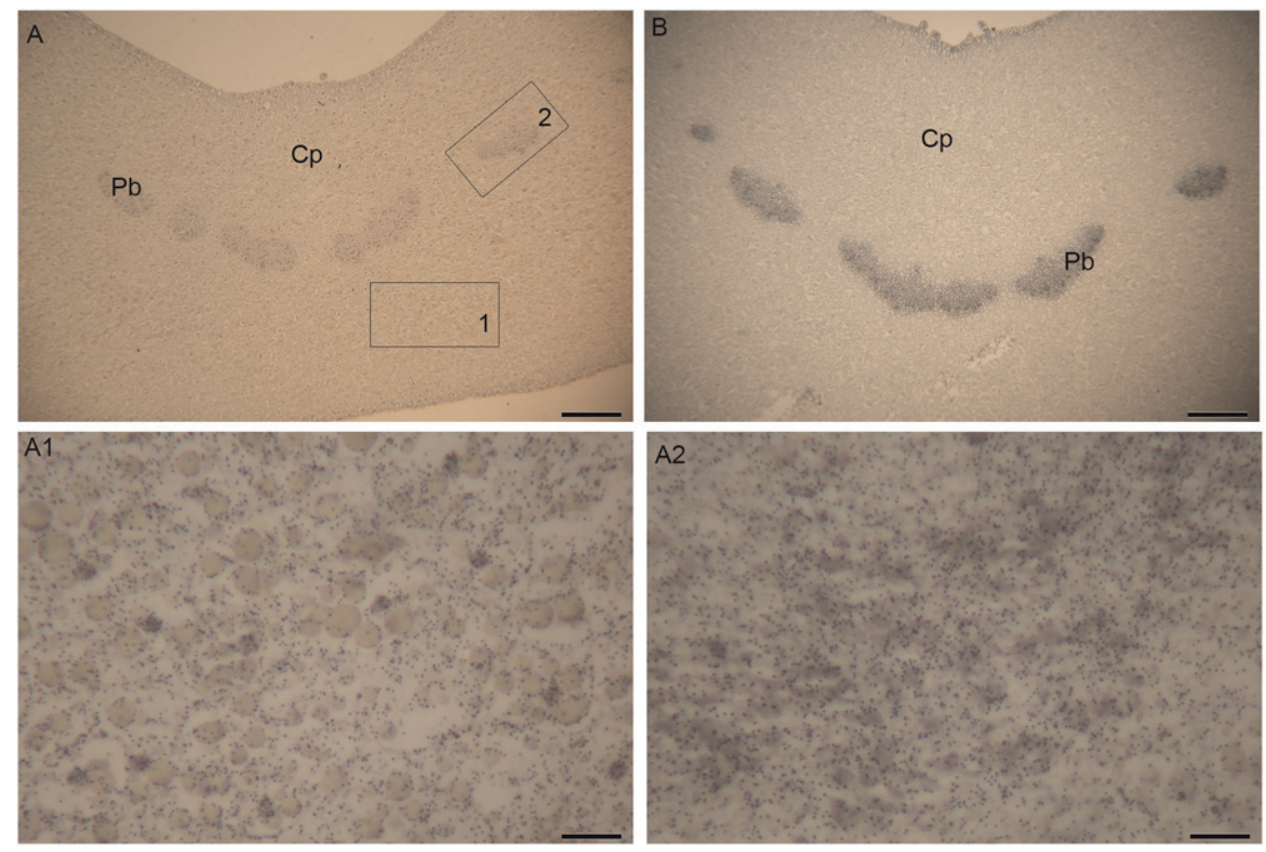

Fig. 2. Immunohistochemical localization of indole-3-acetic acid (IAA) in walnut cotyledon explants at 0 and $24 \mathrm{~h}$ of root induction. The reddish brown silver particles were the IAA signal. (A) Immunolocalization of IAA in walnut cotyledon explants before root induction. IAA was distributed throughout the walnut cotyledon explants. (A1) Details of boxed area 1 in A showing silver particles residing in the cotyledonous parenchyma. (A2) Details of boxed area 2 in A showing silver particles residing in the provascular bundles. (B) Immunolocalization of IAA in walnut cotyledon explants at $24 \mathrm{~h}$ of root induction. IAA was mainly distributed in the provascular bundles; $C p=$ cotyledonous parenchyma; $P b=$ provascular bundle. Bars: $(\mathbf{A}-\mathbf{B}) 100 \mu \mathrm{m},(\mathbf{A 1}-\mathbf{A 2}) 5 \mu \mathrm{m}$. 

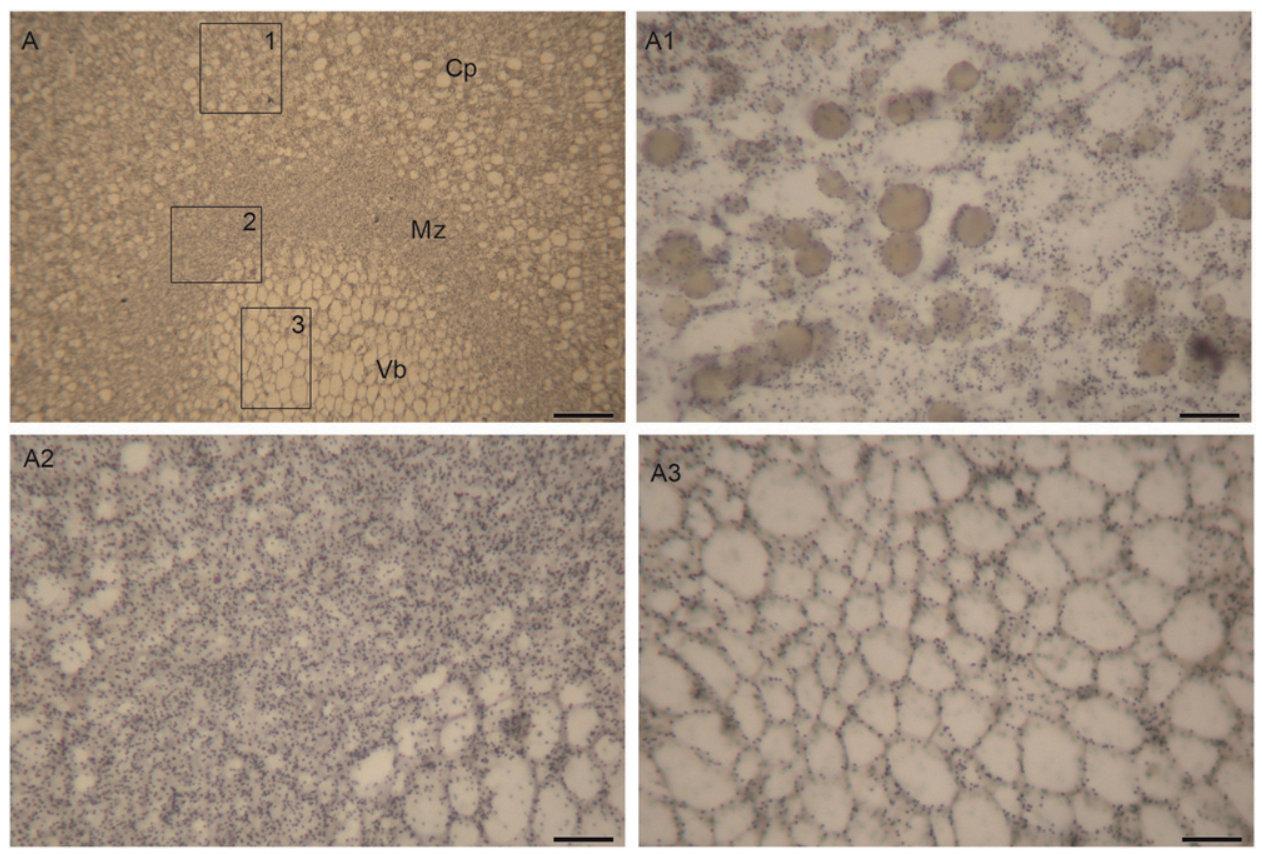

Fig. 3. Immunohistochemical localization of indole-3-acetic acid (IAA) in walnut cotyledon explants at $48 \mathrm{~h}$ of root induction. (A) IAA was distributed in the cotyledonous parenchyma, meristematic zones, and vascular bundles. (A1) Details of boxed area 1 in A showing silver particles residing in the cotyledonous parenchyma. (A2) Details of boxed area 2 in A showing silver particles residing in the annular meristematic zones. (A3) Details of boxed area 3 in A showing silver particles residing in the fully differentiated vascular bundles; $C p=$ cotyledonous parenchyma; $V b=$ vascular bundle; $M z=$ meristematic zone. Bars: (A) $50 \mu \mathrm{m}$, (A1-A3) $5 \mu \mathrm{m}$.
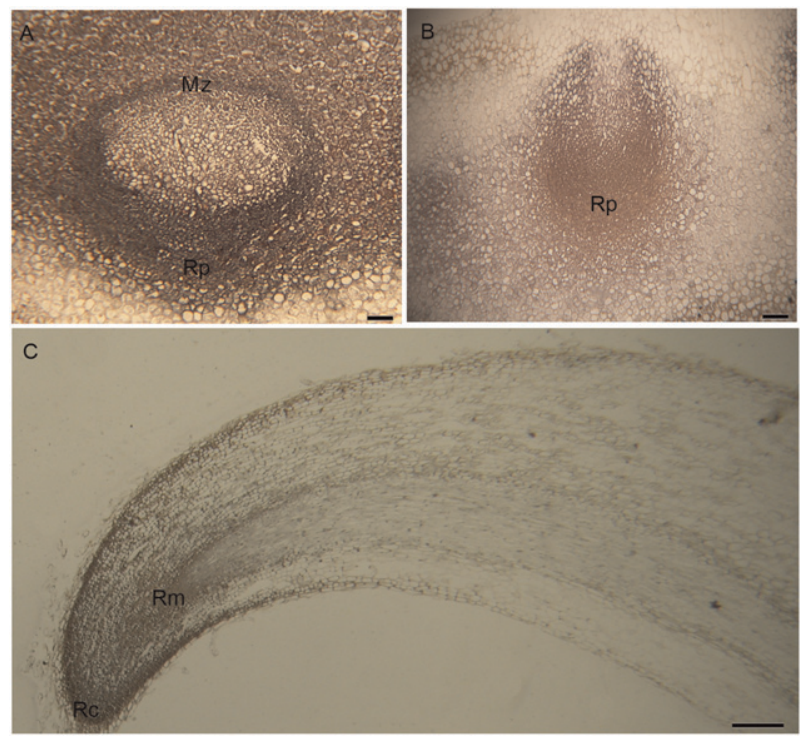

Fig. 4. Immunohistochemical localization of indole-3-acetic acid (IAA) in walnut cotyledon explants at 72 and $96 \mathrm{~h}$ of root induction and in the adventitious root. (A) Immunolocalization of IAA in walnut cotyledon explants at $72 \mathrm{~h}$ of root induction. IAA was mainly distributed in the annular meristematic zones and root primordia. (B) Immunolocalization of IAA in the walnut cotyledon explants at $96 \mathrm{~h}$ of root induction. IAA was mainly distributed in the root primordia. (C) Immunolocalization of IAA in adventitious root from walnut cotyledon explants. IAA was mainly distributed in the root cap and root meristem; $M z=$ meristematic zone; $R p=$ root primordium; $R m=$ root meristem; $R c=$ root cap. Bars: $(\mathbf{A}-\mathbf{B}) 20$ $\mu \mathrm{m},(\mathbf{C}) 100 \mu \mathrm{m}$. than in cotyledonous parenchyma, but this difference was not statistically significant at $P>0.05$ (Fig. 5).

StAGE 1: DIFFERENTIATION OF THE PROVASCULAR BUNDLES (AT 24 H). As the differentiation of provascular bundles progressed, the IAA signal gradually concentrated in the provascular bundles (Fig. 2B). The density of silver particles in the provascular bundles was 206.9/100 $\mu \mathrm{m}^{2}$, higher than in cotyledonous parenchyma $(P<0.01)$ (Fig. 5).

STAGE 2: FORMATION OF ANNULAR MERISTEMATIC ZONES AROUND VASCULAR BUNDLES (AT $48 \mathrm{H}$ ). After $48 \mathrm{~h}$ of culture, annular meristematic zones were formed around the fully differentiated vascular bundles. At that time, the IAA signal was mainly distributed in the meristematic zones and decreased in vascular bundles and cotyledonous parenchyma (Figs. 3 and 5). In the fully differentiated vascular bundles, the IAA was mainly localized on the periphery of the cells (Fig. 3A3).

STAGE 3: RADIALLY ASYMMETRICAL INITIATION OF ADVENTITIOUS ROOT PRIMORDIAL (AT 72 H). After $72 \mathrm{~h}$ of induction, adventitious root primordia were initiated within the annular meristematic zones around vascular bundles. Here, IAA was detected in the meristematic zones and root primordia (Fig. 4A). The signal was stronger in the root primordia than in the meristematic zones $(P<0.01)$ (Fig. 5).

STAGE 4: FORMATION OF FULLY DEVELOPED ADVENTITIOUS ROOT PRIMORDIAL (AT 96 H). After $96 \mathrm{~h}$ of culture, the root primordia were fully developed. At that time, the IAA signal became more localized in the fully developed root primordia (Fig. 4B). The density of silver particles in the root primordia was 284.7/100 $\mu \mathrm{m}^{2}$, higher than in other tissues $(P<0.01)$ (Fig. 5).

Stage 5: formation of adventitious roots (at $144 \mathrm{~h}$ ). Adventitious roots were visible after $144 \mathrm{~h}$ of culture. In adventitious roots, IAA signal was mainly localized in the root cap and root meristem (Fig. 4C).

\section{Analysis of indole-3-acetic acid distribution for each tissue of the cotyledon during adventitious root formation}

In cotyledonous parenchyma, the IAA level showed a dramatic decrease from 0 to $24 \mathrm{~h}(P<0.01)$, then remained stable from 24 to $72 \mathrm{~h}(P>0.05)$, and decreased sharply at $96 \mathrm{~h}(P<$ 0.01 ) (Fig. 6). In the vascular bundle, the IAA level showed a significant increase from 0 to $24 \mathrm{~h}(P<0.01)$, and decreased sharply at $48 \mathrm{~h}(P<0.01)$, then remained stable from 48 to $96 \mathrm{~h}$ $(P>0.05)$ (Fig. 6). The IAA level in meristematic zones showed an increase from 48 to $72 \mathrm{~h}$, but the change was not statistically significant $(P>0.05)$ (Fig. 6). The IAA level in root primordia was stable from 24 to $48 \mathrm{~h}(P>0.05)$ (Fig. 6).

\section{Controls for immunohistochemical localization of \\ indole-3-acetic acid}

The sections of the root primordia and adventitious roots were used as controls. Little IAA signal was detected when 
primary antibody was omitted (Fig. 7A), EDC pre-fixation was omitted (Fig. 7B), or the primary antibody was substituted with normal mouse serum (Fig. 7C). These results indicated that the immunohistochemical localization technique is reliable and the antibody is highly specific.

\section{Discussion}

SPECIFICITY OF THE PRIMARY ANTIBOdIES. The primary antibodies obtained from Agdia were raised against IAABSA conjugated (IAA-17-II-A) and have been used previously to localize endogenous IAA in many studies (Hou and Huang, 2004, 2005; Thomas et al., 2002) and its crossreactivity against 34 IAA-related compounds has been analyzed (Mertens et al., 1985). The crossreaction of the antibodies with IAA analogs such as indole-3-butyric acid and indole-3-acetyl-myo-inositol ester was only $1.3 \%$ and $0.2 \%$, respectively, and with IAA precursors such as tryptophan was also only slightly reactive (less than $0.1 \%$ ). Furthermore, all of the control stainings tested

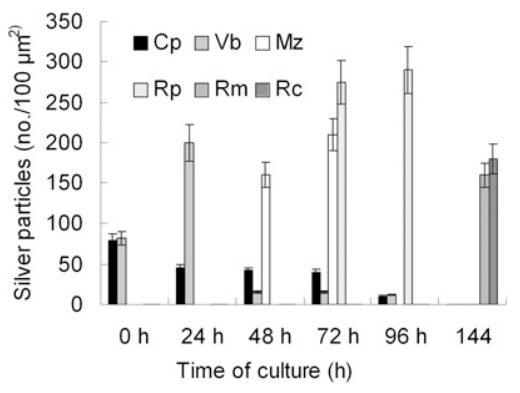

Fig. 5. Density of silver particles in different tissues of the walnut cotyledon explants during adventitious root formation. Values are expressed as the mean number of silver particles per $100 \mu \mathrm{m}^{2} \pm \mathrm{SE} ; C p=$ cotyledonous parenchyma; $\mathrm{Vb}=$ vascular bundle; $\mathrm{Mz}=$ meristematic zone; $R p=$ root primordium; $R m=$ root meristem; $R c=$ root cap. showed little or no signal (Fig. 7). Therefore, the antibodies used in this study were highly specific for IAA.

INDOLE-3-ACETIC ACID DISTRIBUTION COINCIDES WITH VASCULAR DIFFERENTIATION. Vascular differentiation is related to auxin flux (Aloni, 1995, 2001). Canalization of auxin flow has been proposed to explain the pattern of vascular differentiation (Sachs, 1981). The canalization hypothesis proposes a positive feedback mechanism: a proposed gradual restriction of IAA flow from a general field to specialized files of cells, resulting in provascular and later vascular differentiation. Many studies on the relationship between IAA and vascular differentiation have been carried out on leaves, stems, and embryos of plants (Aloni, 1995, 2001; Avsian-Kretchmer et al., 2002; Basu et al., 2002; Sieburth, 1999), but there are few reports on that from fresh cotyledon. In the present study, the IAA signal was distributed throughout the freshly excised cotyledons (Fig. 2A). As the differentiation of provascular bundles progressed, the IAA signal was gradually concentrated in the provascular bundles of the cotyledons (Fig. 2B). These results suggest the accumulation of IAA in the provascular bundles induces provascular, and later vascular differentiation, which is in agreement with the canalization hypothesis (Sachs, 1981). When the provascular bundles were fully differentiated, IAA signal in the vascular bundles decreased and became localized mainly on the periphery of the cells (Fig. 3A3). This distribution pattern in the fully differentiated vascular bundles is interesting and requires further study to reveal the cause.

INDOLE-3-ACETIC ACID DISTRIBUTION DURING THE ADVENTITIOUS ROOT FORMATION. Figure 4A shows that initiation of the root primordia takes place within the annular meristematic zones. Our observation that IAA accumulates strongly in the annular meristematic zones (Figs. 3 and 4A) implies that IAA is responsible for the formation of root primordia within the annular meristematic zones. In contrast to the radially symmetrical distribution of IAA in the annular meristematic zone (Figs. 3 and 4A), radially asymmetrical adventitious root primordia were formed exclusively at the side of the meristematic zone (Fig. 4A-B). These findings suggest that factors other than the IAA are involved in adventitious root formation from cotyledon explants of walnut, which is consistent with the hypothesis that auxin is not the sole signal for lateral root formation (Celenza et al., 1995). When some cells of meristematic zones dedifferentiate into visible root primordia, a strong IAA signal became localized in the root primordia (Fig. 4B). This result differs from previous studies, which reported transient increases in IAA concentration followed by decreases before rooting (Blakesley et al., 1991; De Klerk et al., 1999). In adventitious roots, IAA signal was mainly distributed in the root meristem and root cap (Fig. $4 \mathrm{~F}$ ), which corroborates the results of Ljung et al. (2005) who identified an important auxin source in the meristematic region of the primary root tip. More recently, Petersson et al. (2009) revealed the presence of IAA 


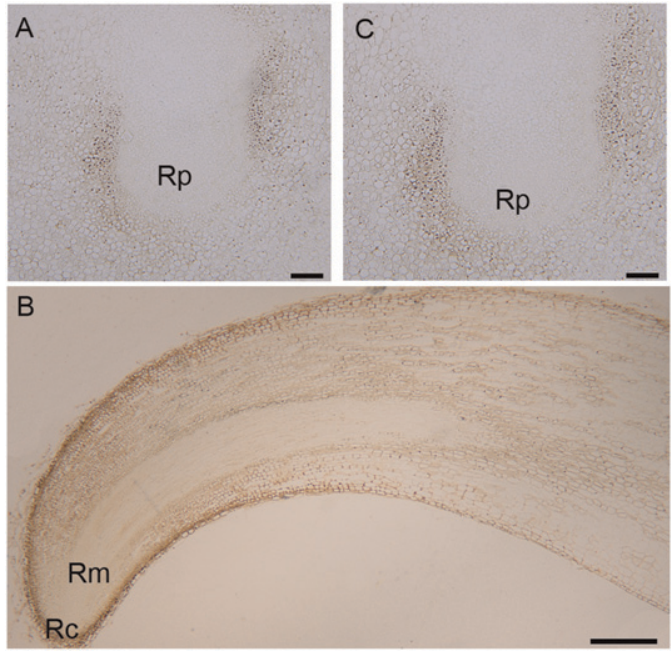

Fig. 7. Controls for the indole-3-acetic acid (IAA) immunolocalization technique. (A) Transverse section of the walnut root primordium stained without 1-ethyl-3-(3-dimethylaminopropyl)-carbodiimide (EDC) pre-fixation. (B) Longitudinal section of the walnut adventitious root stained without the primary IAA antibody. (C) Transverse section of the root primordium stained using normal mouse serum instead of primary antibody. There was little IAA signal detected in any of the control sections; $R p=$ root primordium; $R m=$ root meristem; $R c=$ root cap. Bars: $(\mathbf{A}, \mathbf{C}) 20 \mu \mathrm{m}$, (B) $100 \mu \mathrm{m}$.

concentration gradients within the arabidopsis root tip with a distinct maximum in the organizing quiescent center of the root apex.

In conclusion, this study shows for the first time the precise distribution of IAA during the formation of adventitious roots from walnut cotyledon explants. Our observation that IAA accumulates in specific areas under culture conditions favorable to the induction of cell division and subsequent adventitious root formation suggests a correlation between accumulation of IAA and the induction of either cellular proliferation or determination of developmental fate. The results presented here provide supporting evidence for the concept that signals other than IAA are involved in adventitious root formation. This study also provides a substantial base for further research on specific sites of IAA production and its route of transport during adventitious root formation.

\section{Literature Cited}

Ahmad, N., I. Siddique, and M. Anis. 2006. Improved plant regeneration in Capsicum annuum L. from nodal segments. Biol. Plant. 50:701-704. Aloni, R. 1995. The induction of vascular tissues by auxin and cytokinin, p. 531-546. In: Davies, P.J. (ed.). Plant hormones: Physiology, biochemistry and molecular biology. Kluwer Academic Publishers, Dordrecht, The Netherlands.

Aloni, R. 2001. Foliar and axial aspects of vascular differentiation: Hypotheses and evidence. J. Plant Growth Regul. 20:22-34.

Aloni, R., K. Schwalm, M. Langhans, and C.I. Ullrich. 2003. Gradual shifts in sites of free-auxin production during leaf-primordium development and their role in vascular differentiation and leaf morphogenesis in Arabidopsis. Planta 216:841-853.

Avsian-Kretchmer, O., J.C. Cheng, L. Chen, E. Moctezuma, and Z.R. Sung. 2002. Indole acetic acid distribution coincides with vascular differentiation pattern during Arabidopsis leaf ontogeny. Plant Physiol. 130:199-209.

Basu, S., H. Sun, L. Brian, R.L. Quatrano, and G.K. Muday. 2002. Early embryo development in Fucus distichus is auxin sensitive. Plant Physiol. 130:292-302.

Blakesley, D., G.D. Weston, and J.F. Hall. 1991. The role of endogenous auxin in root initiation. Part 1: Evidence from studies on auxin application, and analysis of endogenous levels. J. Plant Growth Regul. 10:341-353.

Celenza, J.L., Jr., P.L. Grisafi, and G.R. Fink. 1995. A pathway for lateral root formation in Arabidopsis thaliana. Genes Dev. 9:2131-2142.

Chen, D., Y. Ren, Y. Deng, and J. Zhao. 2010. Auxin polar transport is essential for the development of zygote and embryo in Nicotiana tabacum L. and correlated with ABP1 and PM H+-ATPase activities. J. Expt. Bot. 61:1853-1867.

Cooper, W.C. 1935. Hormones in relation to root formation on stem cuttings. Plant Physiol. 10:789-794.

De Klerk, G.J., W. Van der Krieken, and J.C. De Jong. 1999. Review the formation of adventitious roots: New concepts, new possibilities. In Vitro Cell. Dev. Biol. Plant 35:189-199.

Ermel, F.F., S. Vizoso, J.P. Charpentier, C. Jay-Allemand, A.M. Catesson, and I. Couee. 2000. Mechanisms of primordium formation during adventitious root development from walnut cotyledon explants. Planta 211:563-574.

Gangopadhyay, M., D. Chakraborty, S. Dewanjee, and S. Bhattacharya. 2010. Clonal propagation of Zephyranthes grandiflora using bulbs as explants. Biol. Plant. 54:793-797.

Gutmann, M., J.P. Charpentier, P. Doumas, and C. Jay-Allemand. 1996. Histological investigation of walnut cotyledon fragments for a better understanding of in vitro adventitious root initiation. Plant Cell Rep. 15:345-349.

Holgate, C.S., P. Jackson, P.N. Cowen, and C.C. Bird. 1983. Immunogold-silver staining: New method of immunostaining with enhanced sensitivity. J. Histochem. Cytochem. 31:938-944.

Hou, Z.X. and W.D. Huang. 2004. Immunohistochemical localization of IAA in developing strawberry fruit. J. Hort. Sci. Biotechnol. 79:693-698.

Hou, Z.X. and W.D. Huang. 2005. Immunohistochemical localization of IAA and ABP1 in strawberry shoot apexes during floral induction. Planta 222:678-687.

Jay-Allemand, C., V. De Pons, P. Doumas, P. Capelli, L. Sossountzov, and D. Cornu. 1991. Formation de racines in vitro à partir de cotylédons de noix (Juglans sp.): Un modèle d'étude de la rhizogénèse chez les espèces ligneuses. Comptes rendus de l'Académie des sciences 312:369-375.

Ljung, K., A.K. Hull, J. Celenza, M. Yamada, M. Estelle, J. Normanly, and G. Sandberg. 2005. Sites and regulation of auxin biosynthesis in Arabidopsis roots. Plant Cell 17:1090-1104.

Ludwig-Muller, J., A. Vertocnik, and C.D. Town. 2005. Analysis of indole-3-butyric acid-induced adventitious root formation on Arabidopsis stem segments. J. Expt. Bot. 56:2095-2105.

Mertens, R., J. Eberle, A. Arnscheidt, A. Ledebur, and E.W. Weiler. 1985. Monoclonal antibodies to plant growth regulators. II. Indole-3acetic acid. Planta 166:389-393.

Moctezuma, E. 1999. Changes in auxin patterns in developing gynophores of the peanut plant (Arachis hypogaea L.). Ann. Bot. (Lond.) 83:235-242.

Moctezuma, E. and L.J. Feldman. 1999. Auxin redistributes upwards in graviresponding gynophores of the peanut plant. Planta 209:180-186.

Nourissier, S. and O. Monteuuis. 2008. In vitro rooting of two Eucalyptus urophylla $\times$ Eucalyptus grandis mature clones. In Vitro Cell. Dev. Biol. Plant 44:263-272.

Petersson, S.V., A.I. Johansson, M. Kowalczyk, A. Makoveychuk, J.Y. Wang, T. Moritz, M. Grebe, P.N. Benfey, G. Sandberg, and K. Ljung. 2009. An auxin gradient and maximum in the Arabidopsis root apex shown by high-resolution cell-specific analysis of IAA distribution and synthesis. Plant Cell 21:1659-1668.

Sachs, T. 1981. The control of the patterned differentiation of vascular tissues. Adv. Bot. Res. 9:151-262.

Shi, L., I. Miller, and R. Moore. 1993. Immunocytochemical localization of indole-3-acetic acid in primary roots of Zea mays. Plant Cell Environ. 16:967-973.

Sieburth, L.E. 1999. Auxin is required for leaf vein pattern in Arabidopsis. Plant Physiol. 121:1179-1190.

Thomas, C., R. Bronner, J. Molinier, E. Prinsen, H. van Onckelen, and G. Hahne. 2002. Immuno-cytochemical localization of indole-3-acetic acid during induction of somatic embryogenesis in cultured sunflower embryos. Planta 215:577-583. 